\title{
Study Methodology: Crux of a Research Article
}

\author{
Debajyoti Mohanty • Anjay Kumar • \\ Ashwani Kumar Dalal
}

Received: 8 October 2013 / Accepted: 18 November 2013/Published online: 28 November 2013

(C) Association of Surgeons of India 2013

We have read the article "A prospective randomized study comparing non-absorbable polypropylene (Prolene ${ }^{\mathbb{B}}$ ) and delayed absorbable polyglactin 910 (Vicryl $\left.{ }^{\circledR}\right)$ suture material in mass closure of vertical laparotomy wounds" by Pandey et al. [1] with interest. The authors had analyzed the two different types of suture materials in 100 patients randomly allocated to each intervention arm in terms of postoperative wound dehiscence.

The inclusion and exclusion criteria of a study need to be stringent so that only the patients satisfying the predefined characteristics are enrolled and studied. This study had included five patients of obstructed umbilical hernia while listing presence of abdominal hernia as one of the exclusion criteria. The authors had included 22 patients with hemoperitoneum and 20 patients of blunt abdominal trauma. Blunt abdominal trauma is considered the leading cause of hemoperitoneum mandating emergency laparotomy. Whether hemoperitoneum in the 22 patients was secondary to blunt abdominal trauma or some other pathology needs to be specified. Further, estimation of sample size for this study on the basis of the primary outcome measure of postoperative wound dehiscence could have enlightened us about the precision of the observed results.

Randomized trials are the current gold standard to compare the effect of different interventions. Proper outlining of the technique of randomization along with the method of blinding employed improves the credibility of a study. The authors had excluded 11 randomized patients from analysis as they were not explored via vertical laparotomy incision. Ideally, these patients should not have been enrolled for randomization at the first place as the defined inclusion criteria was patients undergoing elective or emergency midline laparotomy. At the

D. Mohanty $(\varangle) \cdot$ A. Kumar $\cdot$ A. K. Dalal

Department of General Surgery, All India Institute of Medical

Sciences, Raipur, Chhattisgarh 492099, India

e-mail: debajyoti.mohanty@gmail.com same time, 13 patients were not included in randomization due to use of a different type of suture material. This creates confusion regarding the timing of randomization technique. Whether randomization was carried out at the time of admission, at the time of making the surgical incision, or at the time of abdominal fascial closure needs to be clarified.

The study mentioned of increased incidence of wound dehiscence in the patients undergoing emergency surgery. This assumption was not supported with documentary evidence as we are unable to find any data regarding the incidence of wound dehiscence following emergency surgery in the "Results." The increased incidence can be due to the simple fact that majority of included patients had undergone emergency intervention. Poor general condition of patients, malnutrition, increased risk of surgical field contamination, and surgeon fatigue are the known risk factors for the increased incidence of wound complication in emergency situations [2]. The authors had listed lack of proper sterilization in the emergency setup as one of the factors for this increased incidence. We believe that in this modern era of health-care delivery system, the practice of standard sterilization protocols should not be denied to the patients undergoing emergency surgery.

Lastly, disclosure about the source of funding is essential in this article as the authors had chosen to incorporate the brand name of the suture materials in their work.

\section{References}

1. Pandey S, Singh M, Singh K, Sandhu S (2013) A prospective randomized study comparing non-absorbable polypropylene (Prolene ${ }^{\text {B }}$ ) and delayed absorbable polyglactin $910\left(\right.$ Vicryl $^{18}$ ) suture material in mass closure of vertical laparotomy wounds. Indian J Surg 75(4):306-310

2. van Ramshorst GH, Nieuwenhuizen J, Hop WC, Arends P, Boom J, Jeekel J, Lange JF (2010) Abdominal wound dehiscence in adults: development and validation of a risk model. World J Surg 34(1):20-27 